\title{
THE FEBRUARY MEETING IN TUCSON
}

The five hundred sixty-fifth meeting of the American Mathematical Society was held at the University of Arizona, Tucson, Arizona, on Thursday, Friday, and Saturday, February 18-20, 1960. There were 75 registrants at this meeting, including 68 members of the Society.

By invitation of the Committee to Select Hour Speakers for Far Western Sectional Meetings and with the financial support of the National Science Foundation, the sessions on Thursday and Friday were devoted to a Symposium on Differential Geometry. The Program Committee for this Symposium consisted of Professor C. B. Allendoerfer, Chairman, Professors Herbert Busemann, Hans Samelson, and D. C. Spencer. Professor Allendoerfer presided at the Thursday morning session of the Symposium which consisted of an hour address by Professor Raoul Bott, Harvard University, entitled $A$ report on the unitary group, followed by three shorter talks: On compact, Riemannian manifolds with constant, negative curvature, by Professor Eugenio Calabi, University of Minnesota, Elementary remarks on surfaces with curvature of fixed sign, by Professor Louis Nirenberg, New York University, and Canonical forms on frame bundles of higher order contact by Professor Shoshichi Kobayashi, University of British Columbia. Professor Samelson was the chairman of the Thursday afternoon session. The principal hour address was Some remarks on homological analysis and structures by Professor D. C. Spencer, Princeton University. The shorter addresses were Almost product structures by Professor A. G. Walker, University of Liverpool and University of Washington, Differential-geometric methods in the study of families of complex structures, Professor Albert Nijenhuis, University of Washington, and Complex homogeneous contact manifolds, by Professor W. M. Boothby, Washington University. Professor Spencer was the chairman of the session on Friday morning. This session included an hour address entitled Vector bundles and homogeneous spaces by Professor F. E. P. Hirzebruch, University of Bonn and the Institute for Advanced Study, and the following shorter papers: Algebraic homogeneous spaces and the cohomology of nilpotent Lie algebras, Professor Bertram Kostant, University of California, Berkeley, Locally affine spaces and uniform subgroups of Lie groups, Professor Louis Auslander, Indiana University, and The cohomology of Lie rings and the lifting of group actions to fibre bundles, Dr. R. S. Palais, Institute for Advanced Study. The final session of the Symposium on Friday 
afternoon was under the chairmanship of Professor Busemann. The principal hour address was given by Professor John Milnor of Princeton University and the University of California, Berkeley. It was entitled $A$ procedure of killing homotopy groups of differential manifolds. This was followed by three shorter addresses: The AlexanderPontrjagin duality in function space, Professor James Eells, Jr., Columbia University, Homology of principal bundles, Dr. R. K. Lashof, University of Chicago, and On immersion of manifolds, by Professor Hans Samelson, University of Michigan.

The Proceedings of this Symposium will be published in book form by the Society at an early date.

By invitation of the Committee to Select Hour Speakers for Far Western Sectional Meetings, Professor T. S. Motzkin of the University of California at Los Angeles addressed the Society Saturday morning on Convex sets in analysis. He was introduced by Professor John Todd. There were three sessions for contributed papers with Professor F. B. Fuller, Professor Ivan Niven and Dr. J. L. Selfridge presiding.

R. S. PIERCE, Associate Secretary 